\title{
Correction to: Motif dynamics in signed directional complex networks
}

\author{
Youngjai Park ${ }^{1} \cdot$ Mi Jin Lee ${ }^{1}$ Seung-Woo Son ${ }^{1,2}$
}

Published online: 9 April 2021

(c) The Korean Physical Society 2021

\section{Correction to: Journal of the Korean Physical Society (2021) 78:535-541 \\ https://doi.org/10.1007/s40042-021-00058-6}

Due to an unfortunate oversight, the correspondence author of this article has been given erroneously. Corresponding authors of this paper should be changed to Seung Woo Son and Mi Jin Lee.

Publisher's Note Springer Nature remains neutral with regard to jurisdictional claims in published maps and institutional affiliations.

The original article can be found online at https://doi.org/10.1007/ s40042-021-00058-6.

Mi Jin Lee

mijinlee@ hanyang.ac.kr

$\triangle$ Seung-Woo Son

sonswoo@hanyang.ac.kr

1 Department of Applied Physics, Hanyang University, Ansan 15588, Korea

2 Asia Pacific Center for Theoretical Physics (APCTP), Pohang 37673, Korea 